\title{
A phase II randomized trial comparing radiotherapy with concurrent weekly cisplatin or weekly paclitaxel in patients with advanced cervical cancer
}

\author{
Fady B Geara ${ }^{1 *}$, Ali Shamseddine ${ }^{2}$, Ali Khalil ${ }^{3}$, Mirna Abboud ${ }^{1}$, Maya Charafeddine ${ }^{2}$, Muhieddine Seoud ${ }^{3}$
}

\begin{abstract}
Purpose/Objective: This is a prospective comparison of weekly cisplatin to weekly paclitaxel as concurrent chemotherapy with standard radiotherapy for locally advanced cervical carcinoma.

Materials/Methods: Between May 2000 and May 2004, 31 women with FIGO stage IB2-IVA cervical cancer or with postsurgical pelvic recurrence were enrolled into this phase II study and randomized to receive on a weekly basis either $40 \mathrm{mg} / \mathrm{m}^{2}$ Cisplatin (group l; 16 patients) or $50 \mathrm{mg} / \mathrm{m}^{2}$ paclitaxel (group II; 15 patients) concurrently with radiotherapy. Median total dose to point A was 74 Gy (range: 66-92 Gy) for group I and 66 Gy (range: 40-98 Gy) for group II. Median follow-up time was 46 months.

Results: Patient and tumor characteristics were similar in both groups. The mean number of chemotherapy cycles was also comparable with $87 \%$ and $80 \%$ of patients receiving at least 4 doses in groups I and II, respectively. Seven patients (44\%) of group I and 8 patients (53\%) of group II developed tumor recurrence. The Median Survival time was not reached for Group I and 53 months for group II. The proportion of patients surviving at 2 and 5 years was $78 \%$ and $54 \%$ for group I and $73 \%$ and $43 \%$ for group II respectively.
\end{abstract}

Conclusions: This small prospective study shows that weekly paclitaxel does not provide any clinical advantage over weekly cisplatin for concurrent chemoradiation for advanced carcinoma of the cervix.

\section{Introduction}

In many developing countries, cervical cancer remains a major public health problem with high overall incidence and higher frequency of advanced stage at diagnosis. Radiation therapy remains the main treatment modality for patients with advanced cervical cancer the results of which depend on disease stage, tumor volume, presence of involved lymph nodes, delivered radiation dose, treatment duration, hemoglobin level, and the optimal use of intracavitary brachytherapy [1-5]. Nodal involvement, particularly of paraaortic nodes, was reported to be the most important adverse prognostic factor, reducing survival by one-half. A series of controlled randomized

\footnotetext{
* Correspondence: fg00@aub.edu.lb

'Department of Radiation Oncology, The American University of Beirut Medical Center, Bliss Street, Beirut, Lebanon

Full list of author information is available at the end of the article
}

studies have shown that the outcome of these patients can be improved by the use of concurrent chemoradiotherapy (CTRT) protocols [6-16]. Based on these trials, the National Cancer Institute issued a clinical alert stating that "strong consideration should be given to the incorporation of concurrent cisplatin-based chemotherapy with radiation in women who require radiation therapy for cervical cancer" $[17,18]$. At present, the integration of radiosensitizing cisplatin-based chemotherapy with local treatment is considered the accepted standard in the management of high-risk patients with carcinoma of the cervix [19-21].

Despite the use of concurrent CTRT, many patients continue to fail in the pelvis (20-25\%) and at distant sites (10-20\%), [6,16,21-23]. In addition, the use of cisplatin-based chemotherapy concurrently with RT has not been invariably effective. A study by the National 
Cancer Institute of Canada using weekly concurrent single agent cisplatin has shown no clinical benefit from this schedule [24]. These facts have stimulated interests in exploring other concurrent combinations with potentially more clinical effect. Paclitaxel is a taxane chemotherapy drug that was found to have significant activity in solid tumors especially epithelial ovarian cancer, lung, and breast cancer [25-28]. Preclinical studies have shown a radiosensitizing effect of paclitaxel in human cervical cancer cell lines $[29,30]$. It was also shown that this drug exerts a preferential cytotoxic activity in human cervical cancer cells with low Raf-1 kinase activity which makes it desirable to be used in conjunction with radiotherapy [30]. The clinical feasibility of concurrent RT and paclitaxel was tested in phase I trials and a maximum tolerated dose (MTD) of $50 \mathrm{mg} / \mathrm{m} 2$ per week concurrently with radiation therapy was established $[31,32]$. In addition, the clinical efficacy of paclitaxel has been tested in phase II and III studies for metastatic and recurrent cervical cancer with objective response rates ranging between 36 and $47 \%$ [33-35].

In this study, we examine the tumor response, treatment toxicity, and outcome of patients with locally advanced cervical cancer treated by concurrent radiation therapy and chemotherapy using either weekly Cisplatin or weekly paclitaxel.

\section{Methods and materials Patients}

Patients presenting to the American University of Beirut Medical Center, with advanced carcinoma of the cervix, stages IB2-IVA according to the Federation Internationale de Gynecologie Obstetrique (FIGO) staging system, or with measurable central pelvic recurrence, were eligible to enroll in this phase II randomized prospective study. Inclusion criteria also included: age $<80$ years; Gynecologic Oncology Group (GOG) performance status of 0-3; adequate hematological and biochemical profile with absolute neutrophil count $>1.5 \times 10^{9} / \mathrm{L}$, platelets $>100 \times 10^{9} / \mathrm{L}$; creatinine $<1.5$, liver enzymes (AST and ALT) $<3 \times$ normal, and bilirubin $<1.25$ normal. Patients with evidence of enlarged paraaortic lymphnodes, history of peripheral neuropathy, prior radiotherapy, prior chemotherapy (neoadjuvant), hypersensitivity to cisplatin or paclitaxel, or other synchronous malignancies were considered not eligible. Treatment was started within 48 hours of randomization. Between May 2000 and May 2004, 31 women were enrolled and randomized to receive on a weekly basis either $40 \mathrm{mg} / \mathrm{m}^{2}$ Cisplatin (group I; 16 patients) or 50 $\mathrm{mg} / \mathrm{m}^{2}$ paclitaxel (group II; 15 patients) concurrently with radiotherapy.

\section{Chemotherapy}

Patients were randomized into two groups: group 1 treated with weekly cisplatin and group 2 treated with weekly paclitaxel. Group I patients received weekly cisplatin $40 \mathrm{mg} / \mathrm{m}^{2}$ given intravenously in $200 \mathrm{cc}$ of D5NSS over one hour. Premedication consisted of dexamethasone $8 \mathrm{mg} \mathrm{IV}$, and a 5HT3-receptor antagonist as antiemetic with hydration for two hours before and after chemotherapy with D5-NSS at $150 \mathrm{cc} /$ hour. Group II patients were treated with weekly paclitaxel $50 \mathrm{mg} / \mathrm{m}^{2}$ given intravenously in $500 \mathrm{cc}$ of D5W in a glass container over three hours. Premedication consisted of dexamethasone $8 \mathrm{mg}$ IV, Benadryl $25 \mathrm{mg}$ IV, Ranitidine 50 mg IV, and a 5HT3-receptor antagonist as antiemetic. Planned treatment was for an average of 5-6 cycles to coincide with the duration of external beam radiation and continued during brachytherapy.

\section{Radiation therapy}

Radiation treatment consisted of external beam radiation to $40 \mathrm{~Gy}$ in 20 fractions using $15 \mathrm{MV}$ photons and an anteroposterior pelvic field arrangement. This was followed by low-dose, or high-dose rate uterovaginal brachytherapy (UVB). Patients treated before 2001 received low-dose Cesium brachytherapy, but after 2001, eligible patients were treated with high-dose rate Iridium brachytherapy. Patients who had parametrial involvement also received a parametrial boost to the affected side. Median total dose to point A was 74 Gy (range: 66-92 Gy) for group I and 66 Gy (range: 40-98 Gy) for group II. Patients who had poor vaginal anatomy, or had prior hysterectomy were treated with an external radiation boost instead of uterovaginal brachytherapy to a median total central pelvic dose of 60.4 Gy (range: 60-66 Gy). The first HDR brachytherapy application was inserted no later than 7 days after completion of pelvic radiation.

\section{Baseline evaluation and follow-up}

Tumor size was assessed clinically by two different examiners prior to, and following treatment. Initial work-up included a complete blood and platelet counts $(\mathrm{CBC})$, creatinine $(\mathrm{Cr})$, liver function tests (SGPT, Alk. P., Gamma GT). Computerized tomography (CT) scanning of the abdomen and pelvis, and chest $\mathrm{x}$-ray (CXR). During treatment, patients had weekly $\mathrm{CBC}, \mathrm{Cr}$., and liver function tests. Patients who developed any allergic reactions during cisplatin and paclitaxel, were subsequently pretreated with dexamethasone $8 \mathrm{mg}$ orally every 8 hours for three doses prior to the admission for chemotherapy. After completing therapy, patients had a repeat baseline examination and CT scan of the abdomen and pelvis. Later follow-up evaluation consisted of repeat pelvic examinations every 3 months for the first 
two years and every six months thereafter until progression or deaths. Pap smears, CXR, and CT scans of the abdomen and pelvis were obtained every 6 months.

\section{Endpoints ans statistical anlysis}

The primary endpoints were treatment response, overall survival, and time to relapse. Time to relapse was defined from the date of entry into the study to the date of recurrence. Patients with progressive disease who never achieved complete response, were censored for relapse at the end of radiation therapy, which is approximately 2 months and half from the date of entry. Disease free survival was defined as alive with no evidence of disease at the time of last follow-up or dead without evidence of disease. Survival was calculated from the date of entry to the date of death or last follow-up. Cumulative survival rates was estimated by the KaplanMeier method and compared using the Logrank tests. Chi-square and the student' $t$-test were used for comparative analyses. Statistical significance was defined as $\mathrm{p}<0.05$. Data were analyzed using a SPSS program version 16.0. Patient lost to follow up were censored from the analysis at the time of their last follow up date. Treatment related toxicity was assessed as a secondary endpoint. Adverse events were graded according to the National Cancer Institute Common Toxicity Criteria grading system [36]. The incidence, severity, and causal relation to treatment of these events were compared between treatment groups. The study was conducted according to globally accepted standards of Good Clinical Practice and in agreement with the declaration of Helsinki and was approved by the Institutional Review Board (IRB). All patients have signed an informed consent form before their enrollement into the study.

\section{Results}

Thirty one consecutive patients met the eligibility criteria and were enrolled; 16 patients were randomized to the cisplatin arm (group I) and 15 to the paclitaxel arm (group II). The patients' demographic characteristics are listed in table 1. Age, parity, histology, presence of hydronephrosis, and lymph node involvement were not different in both groups. Only median tumor size was slightly larger for group II patients $(6 \mathrm{~cm})$ compared to that of group I patients $(4.75 \mathrm{~cm})$, but this was not statistically significant $(\mathrm{p}=0.16)$.

Treatment details are listed in table 2. The mean number of chemotherapy cycles was comparable [4.6 \pm 0.9 vs. $4.3 \pm 1.3$ ], with $87 \%$ and $80 \%$ of patients receiving $\geq 4$ doses in groups I and II, respectively. The median dose to point A was slightly higher for group I patients (74 vs $66 \mathrm{~Gy}, \mathrm{p}=0.27$ ). This is due to the fact that more patients in group II were enrolled as pelvic recurrences after surgery and received an external beam
Table 1 Patient characteristics. Group I received concurrent cisplatin and group II received concurrent paclitaxel

\begin{tabular}{lll}
\hline & Group I & Group II \\
\hline Number of patients & $16^{*}$ & $15^{*}$ \\
Median age (years) & $56(37-71) *$ & $48(38-80)^{*}$ \\
Parity & $4(0-11) *$ & $5(2-9) *$ \\
Squamous cell pathology & $13(81 \%)^{*}$ & $14(93 \%)^{*}$ \\
Stage III-IVA & $7(44 \%)^{*}$ & $8(53 \%)^{*}$ \\
Median Tumor size $(\mathrm{cm})$ & $4.75(2.5-8) *$ & $6(3-11)^{*}$ \\
Hydronephrosis & $3(19 \%)^{*}$ & $5(33 \%)^{*}$ \\
Positive pelvic lymph nodes & $3(19 \%)^{*}$ & $5(33 \%)^{*}$ \\
Enrolled as pelvic recurrence & $2(13 \%) *$ & $5(33 \%)^{*}$ \\
\hline
\end{tabular}

* Shown in parentheses, are range or percentage values as applicable.

radiation boost instead of uterovaginal brachytherapy. In group I, only 3 patients (19\%) did not receive UVB compared to 6 patients $(40 \%)$ in group II $(\mathrm{p}=0.84)$. The mean duration of radiation therapy was similar in both groups $(53.1 \pm 9.9$ vs. $55.8 \pm 9.0$ days; $\mathrm{p}=0.56)$. Treatment related acute toxicity is listed in table 3. Both groups had comparable hematological toxicity, but more patients in group II had severe diarrhea (53\% vs. 37\%), and severe allergic reactions ( $40 \%$ vs. $6 \%$ ). In two group II patients, chemotherapy had to be discontinued because of drug-related severe allergic reactions. Also, delay in chemotherapy was more common with group II than with group I patients ( $47 \%$ vs. $25 \%$ ), but this difference was not statistically significant $(\mathrm{p}=0.22)$.

There was a non-significant trend for more local and distant failure in group II. Seven patients of group I (44\%) suffered tumor relapse [three locally and four outside the pelvis], while 8 group II patients (53\%) developed tumor recurrence [three locally, two with distant metastasis, and three locally and distantly]. At 2 years, local control rates were $93 \%$ for group I and $70 \%$ for group II ( $\mathrm{p}=\mathrm{ns})$.

Figure 1 shows progression free survival with no difference for both groups. The Median survival time was

Table 2 Chemotherapy and radiation therapy treatment parameters

\begin{tabular}{lll}
\hline & Group I & Group II \\
\hline Chemotherapy cycles & $5(3-6) *$ & $5(1-6) *$ \\
>4 cycles & $14(87 \%) *$ & $12(80 \%)^{*}$ \\
EBRT dose & $40(40-66)^{*}$ & $40(40-66) *$ \\
Dose to point A & $33(5-52) *$ & $34(0-58) *$ \\
No UV brachytherapy & $3(19 \%) *$ & $6(40 \%)^{*}$ \\
Total dose to point A & $75(60-93) *$ & $66(40-98) *$ \\
\hline
\end{tabular}

Group I received concurrent cisplatin and group || received concurrent paclitaxel.

* Numbers represent number of patients with percentages when indicated, or median values with ranges (shown in parentheses).

$\mathrm{EBRT}=$ external beam radiotherapy; UV=uterovaginal 
Table 3 Incidence and types of acute toxicity

\begin{tabular}{lll}
\hline Toxicity endpoint & Group I & Group II \\
\hline Leucopenia (grade 1-4) & $3(19 \%)$ & $4(27 \%)$ \\
All hematologic (grade 3-4) & $2(12 \%)$ & $1(7 \%)$ \\
Neurologic (grade I) & $2(12 \%)$ & 0 \\
Diarrhea (grade 3-4) & $6(37 \%)$ & $8(53 \%)$ \\
Allergic reactions & $1(6 \%)$ & $6(40 \%)$ \\
Delay in CT & $4(25 \%)$ & $7(47 \%)$ \\
\hline
\end{tabular}

Group I received concurrent cisplatin and group II received concurrent paclitaxel. Two patients in group II had to discontinue treatment in cycles 1 \& 2 because of severe allergic reactions.

One patient in group I developed an allergic reaction to metochlorpropramide given as antiemetic.

not reached for Group I and is 53 months for group II (Figure 2). At the end of the study $54 \%$ of group I $v s$. $42 \%$ of group II patients were still alive. The proportion of patients surviving at 2 and 5 years was $78 \%$ and $54 \%$ for group I $v s .73 \%$ and $42 \%$ for group II respectively ( $\mathrm{p}$ $=0.651$ ).

Several treatment and patient related factors were assessed for their prognostic significance. These included age, number of chemotherapy cycles, disease stage, presence of hydronephrosis, tumor size, delay in chemotherapy, radiation dose to point $\mathrm{A}$, and the use of uterovaginal brachytherapy. None of these factors was found to have significant influence on disease free or overall survival (table 4).

\section{Discussion}

This small phase II study provides a direct comparison between cisplatin and paclitaxel used as weekly concurrent chemotherapy with definitive radiation for

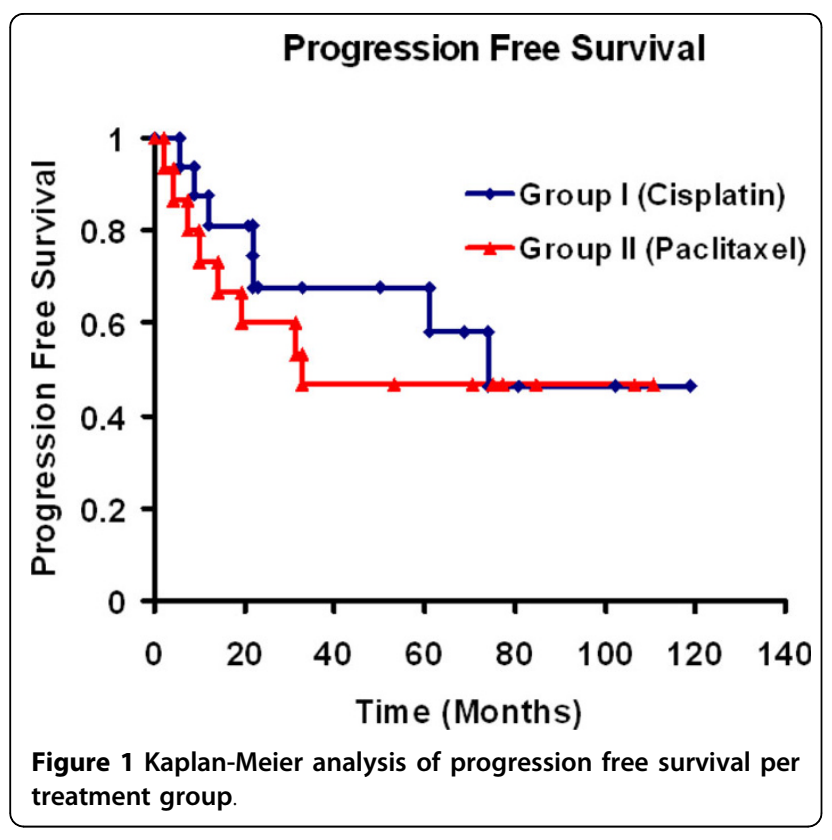

Table 4 Univariate analysis of several patient, tumor, and treatment parameters

\begin{tabular}{ll}
\hline Factor & p-value \\
\hline Age & 0.29 \\
Number of chemotherapy cycles & 0.06 \\
Stage & 0.75 \\
Hydronephrosis & 0.93 \\
Tumor size & 0.16 \\
Delay in Chemotherapy & 0.22 \\
Dose to Point A & 0.27 \\
HDR brachytherapy & 0.84 \\
\hline
\end{tabular}

Only the number of chemotherapy cycles was borderline significant.

advanced carcinoma of the cervix. Our data indicate that the overall response and progression free survival rates with the use of paclitaxel, which is the experimental arm, are not superior to those with cisplatin. In fact, there were non-significant trends for a higher relapse rate, higher gastrointestinal toxicity, and more allergic reactions in the concurrent paclitaxel group. Taken together, these results indicate that paclitaxel does not provide any clinical advantage over the current standard of concurrent cisplatin in CTRT for patients with advanced cervical carcinoma.

Although many prospective studies had shown that CTRT with cisplatin-based chemotherapy clearly improve the outcome of patients with carcinoma of the cervix, many patients treated on these protocols continue to fail in the pelvis and at distant sites $[6,16,22,23]$. In addition, one intergroup study using weekly concurrent cisplatin with radiotherapy for patients with carcinoma of the cervix could not demonstrate a beneficial effect of CTRT over standard RT

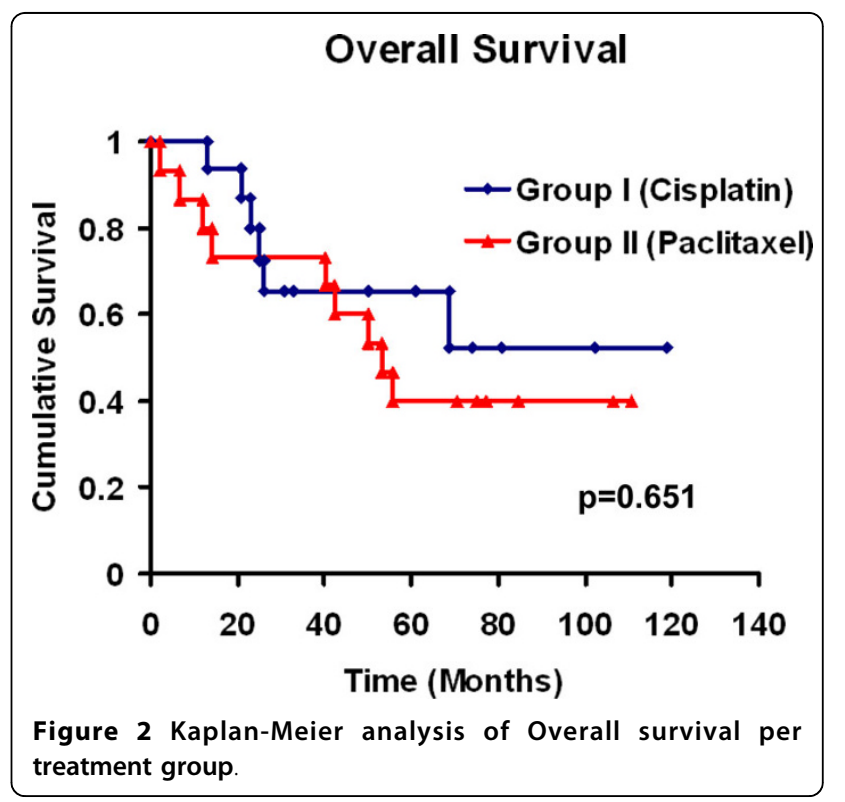


alone [24]. This non-superiority finding was attributed to many factors like possible enrollment of patients with paraaortic lymph nodes, and an imbalance among randomization groups for known prognostic factors such as anemia [22,37]. These facts have lead many groups to investigate other drugs for CTRT like paclitaxel in an attempt to improve on what can be achieved by concurrent cisplatin [31,38-44]. In all these studies paclitaxel was used in conjunction with either cisplatin $(4 / 7 \mathrm{stu}-$ dies) or carboplatin (3/7 studies) but was never used alone for CTRT. The majority of these studies was phase I ( $4 / 7$ studies), with one study being a combined phase I/II study conducted by the GOG [42]. The number of patients enrolled in these studies varied between 8 and 35 patients and the rates of progression free survival ranged between 39 and $88 \%$. The dose limiting toxicity was primarily neutropenia in 4 studies $[38,41,42,44]$ or diarrhea $[31,39,40]$. In our phase II study reported here, we enrolled 31 patients and progression free survival was $61 \%$ for the cisplatin arm and $63 \%$ for the paclitaxel arm with severe grade III diarrhea being the most common toxicity (37\% and $54 \%$ for the cisplatin and paclitaxel groups, respectively). These data are in agreement with what other groups have reported and do not suggest that paclitaxel provides any advantage in outcome or toxicity over the current standard using cisplatin. This finding is in line with what was found in a larger study by the GOG which also compared concurrent single agent CTRT consisting of either weekly cisplatin or protracted 5 -fluorouracil $(5-\mathrm{Fu})$ infusion. The results of that study showed no superiority of the experimental $5-\mathrm{Fu}$ arm and the study was prematurely closed [45].

There are many studies that investigated other experimental protocols for concurrent CTRT in advanced carcinoma of the cervix using various chemotherapy regimens such as 5 -Fu, epirubicin, 5-Fu with mitomycin C, hydroxyurea, gemcitabine, carboplatin, tirapazamine, topotecan, or vinorelbine [46-64]. Few of these drugs were tested in randomized trials like 5-Fu, epirubicin, hydroxyurea, mitomycin and gemcitabine, as single agents or in combination [46-48,53]. Others have only been tested in phase I/II studies and some of them have shown promising results. Perhaps the most promising and most studied drugs of this group are gemcitabine, tirapazamine, and topotecan. In a phase II randomized study by Dueñas-Gonzalez et al, patients with stage IB2IIB disease were randomized to cisplatin or cisplatin plus gemcitabine and concurrent radiation therapy, followed by radical hysterectomy 4 weeks later. The complete pathologic response rate was higher in the cisplatin plus gemcitabine arm compared to the cisplatin alone arm ( $75 \%$ vs. $55 \%$, respectively; $\mathrm{p}=0.02$ ), but gastrointestinal and hematologic toxicities were significantly lower in the cisplatin-alone arm [53]. A phase III randomized trial testing this combination for definitive CTRT in stages IIB to IVA disease, has completed accrual but results are not yet available. Similarly, encouraging results have been obtained in phase I studies using cisplatin-combination CTRT with either topotecan or tirapazamine [60-62]. Several phase I/II studies are currently investigating the combination cisplatin-topotecan and GOG trial 0219 is testing the added value of tirapazamine to cisplatin for CTRT in carcinoma of the cervix.

The rate of gastrointestinal (GI) toxicity in our study manifesting as severe diarrhea, was high in both arms although slightly higher in the paclitaxel arm. In addition there were more severe allergic reactions in the paclitaxel arm and in 2 patients, chemotherapy had to be discontinued due to the severity of these allergic reactions, and in general, more chemotherapy delays were encountered in this group. It is difficult to compare this toxicity pattern with other studies from the literature, because none of these studies used either paclitaxel or cisplatin alone for CTRT, instead they used both drugs in combination with various dose-administration schedules. However, one could note that in at least 3 of the phase I studies that included paclitaxel, severe diarrhea was the limiting toxicity which agrees with our findings $[31,39,40]$. It is of concern that this difference in toxicity between our treatment groups with the disruption and delays in chemotherapy delivery in group II, could have negatively affected this group's outcome. This is particularly important because this group was also at a relative disadvantage regarding tumor bulk (larger median tumor size) and a smaller number of those patients could benefit from UV brachytherapy (9/ 15 vs. 13/16 for group I) due to anatomical constraints. However, because of the small size of the study it was not possible to fully evaluate the influence of these factors either separately or all combined.

A total of 10 patients (32\%) from both treatment groups developed systemic metastases, a rate consistent with what is reported in the literature, and which emphasizes that the risk of distant recurrence remains a major concern for these high risk patients. Taken as individual studies, data from the various CTRT trials have not consistently shown a reduction in distant metastases (DM) in patients receiving systemic chemotherapy when it was primarily given as a radiosensitizer $[6-16,23,24]$. However, when these data were analyzed together, two metaanalyses found a positive effect of concurrent CTRT on distant recurrence $[65,66]$. Among the studies that used platinum-based chemotherapy, only the radiation therapy and oncology group (RTOG) 90-01 study showed a significant effect of CT on the reduction of DM at both 5 and 8 years of follow-up $[7,23]$. It is of interest to note, that in that 
study, chemotherapy was given as full cycles of cisplatin and 5-Fu during the course of RT, and the dose of cisplatin was highest compared to what was used in the other studies $\left(75 \mathrm{mg} / \mathrm{m}^{2}\right.$ vs. 60,50 , or $\left.40 \mathrm{mg} / \mathrm{m}^{2}\right)$. Among the studies with noncisplatin-based chemotherapy, only the study reported by Wong et al. showed a significant impact on DM [14]. In that trial, CT consisted of epirubicin as a single agent for concurrent CTRT followed by adjuvant therapy with the same drug for 5 cycles. It remains unclear what are the key factors that made the experimental arm in these two particular studies effective against DM. One could speculate that the delivery of full cycle and higher doses of chemotherapy, and/or the use of planned adjuvant chemotherapy could, in theory, better address the risk of systemic recurrence. However, this remains speculative until it is demonstrated in controlled randomized studies, and unfortunately, to our knowledge, there are no such studies currently in progress. The only available information comes from retrospective studies and there are some which critically examined this question and reported similar findings. In a single institution study, Kim et al. reported a comparison between 2 well balanced groups of patients with stage IB-II carcinoma of the cervix who were treated by concurrent CTRT with or without 3 additional cycles of adjuvant platinum (cisplatin or carboplatin)-5Fu chemotherapy [67]. The authors found no effect of adjuvant chemotherapy on the incidence of distant metastases or distant nodal relapses. They also found that adjuvant chemotherapy was relatively difficult to complete, with only $63 \%$ of the patients receiving all 3 cycles, and those in the adjuvant group experienced a higher rate of late grade III-IV rectal complications. Similar results were published by Lee et al. on patients receiving adjuvant CTRT after radical hysterectomy and treated either by 3 additional cycles of cisplatin-5Fu or no additional therapy [68]. Although these two studies are not prospective or randomized trials, they still indicate that the routine use of adjuvant cisplatin-based chemotherapy may not be the best approach to address the risk of distant relapse in this patient population.

In summary, these data show that concurrent chemoradiation for advanced cervical cancer using weekly paclitaxel was not superior to concurrent cisplatin and was possibly associated with more severe gastrointestinal toxicity and more allergic reactions. Progression free survival was equivalent with both drugs and failure at distant sites remains high in both groups, which may indicate the need for additional effective therapy.

\footnotetext{
Author details

'Department of Radiation Oncology, The American University of Beirut Medical Center, Bliss Street, Beirut, Lebanon. ${ }^{2}$ Department of Medical
}

Oncology, The American University of Beirut Medical Center, Bliss Street, Beirut, Lebanon. ${ }^{3}$ Department of Obstetrics and Gynecology, Division of Gynecologic Oncology, The American University of Beirut Medical Center, Bliss Street, Beirut, Lebanon.

\section{Authors' contributions}

FG contributed to the study design, patient treatment, evaluation, and was the leading writer of the manuscript. AS, AK, and MA contributed to the study design and manuscript writing and review. MS performed the statistical analysis and contributed to the review. MS is the leading investigator and contributed to patient enrollment, follow-up and to the manuscript writing and review. All authors read and approved the final manuscript.

\section{Competing interests}

The study was partially supported by Bristol Myers Squibb. FG, AS, AK, and MS have received travel funds from Bristol Myers Squibb

Received: 22 May 2010 Accepted: 23 September 2010 Published: 23 September 2010

\section{References}

1. Coia L, Won M, Lanciano R, et al: The Patterns of Care study for cancer of the uterine cervix. Results of the Second National Practice Survey. Cancer 1990, 66:2451-2456.

2. Einhorn N, Trope C, Ridderheim M, et al: A systematic overview of radiation therapy effects in cervical cancer (cervix uteri). Acta Oncol 2003, 42(5-6):546-56.

3. Eifel PJ, Morris M, Wharton JT, et al: The influence of tumor size and morphology on the outcome of patients with FIGO stage IB squamous cell carcinoma of the uterine cervix. Int J Radiat Oncol Biol Phys 1994, 29:9-16.

4. Fyles AW, Pintilie $M$, Kirkbride $P$, et al: Prognostic factors in patients with cervix cancer treated by radiation therapy: results of a multiple regression analysis. Radiother Oncol 1995, 35(2):107-17.

5. Logsdon MD, Eifel PJ: FIGO IIIB squamous cell carcinoma of the cervix: an analysis of prognostic factors emphasizing the balance between external beam and intracavitary radiation therapy. Int J Radiat Oncol Biol Phys 1999, 43:763-75.

6. Keys HM, Bundy BN, Stehman FB, et al: Cisplatin, radiation and adjuvant hysterectomy compared with radiation and adjuvant hysterectomy for Bulky stage IB cervical carcinoma. N Engl J Med 1999, 340:1154-61.

7. Morris M, Eifel PJ, LU J, et al: Pelvic radiation with concurrent chemotherapy compared with pelvic and para-aortic radiation for highrisk cervical cancer. N Engl J Med 1999, 340:1137-43.

8. Kumar JV, Doval DC, Rao R, Rawal S: A retrospective study of patients with locally advanced cancer of the cervix treated with neoadjuvant chemotherapy followed by radical surgery. Int J Gynecol Cancer 2009, 19(3):417-22.

9. Park DC, Suh MJ, Yeo SG: Neoadjuvant paclitaxel and cisplatin in uterine cervical cancer: long-term results. Int J Gynecol Cancer 2009, 19(5):943-7.

10. Umayahara K, Takeshima N, Nose T, Fujiwara K, Sugiyama Y, Utsugi K, Yamashita T, Takizawa K: Phase I study of concurrent chemoradiotherapy with weekly cisplatin and paclitaxel chemotherapy for locally advanced cervical carcinoma in Japanese women. Int J Gynecol Cancer 2009, 19(4):723-7.

11. Tewari KS, Monk BJ: Recent achievements and future developments in advanced and recurrent cervical cancer: trials of the Gynecologic Oncology Group. Semin Oncol 2009, 36(2):170-80.

12. Walker JL, Morrison A, DiSilvestro P, von Gruenigen VE: Gynecologic Oncology Group. A phase I/II study of extended field radiation therapy with concomitant paclitaxel and cisplatin chemotherapy in patients with cervical carcinoma metastatic to the para-aortic lymph nodes: a Gynecologic Oncology Group study. Gynecol Oncol 2009, 112(1):78-84.

13. Rose PG, Bundy BN, Watkins EB, et al: Concurrent Cisplatin-based radiotherapy and chemotherapy for locally advanced cervical cancer. $N$ Engl J Med 1999, 340:1144-53.

14. Wong LC, Ngan HY, Cheung AN, et al: Chemoradiation and adjuvant chemotherapy in cervical cancer. J Clin Oncol 1999, 17(7):2055-60.

15. Peters WA, Liu PY, Barrett RJ, et al: Concurrent chemotherapy and pelvic radiation therapy compared with pelvic radiation therapy alone as 
adjuvant therapy after radical surgery in high-risk early-stage cancer of the cervix. J Clin Oncol 2000, 18(8):1606-13.

16. Whitney CW, Sause W, Bundy BN, et al: Randomized comparison of fluorouracil plus cisplatin versus hydroxyurea as an adjunct to radiation therapy in stage IIB-IVA carcinoma of the cervix with negative paraaortic lymph nodes: a Gynecologic Oncology Group and Southwest Oncology Group study. J Clin Oncol 1999, 17(5):1339-48.

17. National Cancer Institute: Concurrent chemotherapy for cervical cancer. Clinical announcement, Washington, DC. 1999.

18. McNeil C: New standard of care for cervical cancer sets stage for next questions. J Natl Cancer Inst 1999, 91:500a-501a.

19. Eifel PJ: Concurrent chemotherapy and radiation therapy as the standard of care for cervical cancer. Nat Clin Pract Oncol 2006, 3(5):248-55.

20. Thomas GM: Improved treatment for cervical cancer-Concurrent Chemotherapy and radiotherapy. N Engl J Med 1999, 340:1198-1200.

21. Thomas GM: Concurrent chemotherapy and radiation for locally advanced cervical cancer: the new standard of care. Semin Radiat Oncol 2000, 10(1):44-50.

22. Rose PG: Concurrent chemoradiation for locally advanced carcinoma of the cervix: where are we in 2006? Ann Oncol 2006, 17(Suppl 10): x224-9.

23. Eifel PJ, Winter K, Morris M, et al: Pelvic irradiation with concurrent chemotherapy versus pelvic and para-aortic irradiation for high-risk cervical cancer: an update of radiation therapy oncology group trial (RTOG) 90-01. J Clin Oncol 2004, 22(5):872-80.

24. Pearcey $R$, Brundage $M$, Drouin $P$, et al: Phase III trial comparing radical radiotherapy with and without cisplatin chemotherapy in patients with advanced squamous cell cancer of the cervix. J Clin Oncol 2002, 20(4):966-72.

25. Henderson IC, Berry DA, Demetri GD, et al: Improved outcomes from adding sequential Paclitaxel but not from escalating Doxorubicin dose in an adjuvant chemotherapy regimen for patients with node-positive primary breast cancer. J Clin Oncol 2003, 21(6):976-83.

26. McGuire WP, Hoskins WJ, Brady MF, et al: Cyclophosphamide and cisplatin compared with paclitaxel and cisplatin in patients with stage III and stage IV ovarian cancer. N Engl J Med 1996, 334(1):1-6.

27. Belani $\mathrm{CP}$, Choy H, Bonomi $\mathrm{P}$, et al: Combined chemoradiotherapy regimens of paclitaxel and carboplatin for locally advanced non-smallcell lung cancer: a randomized phase II locally advanced multi-modality protocol. J Clin Oncol 2005, 23(25):5883-91.

28. Strauss GM, Herndon J, Maddaus MA, et al: Randomized clinical trial of adjuvant chemotherapy with paclitaxel and carboplatin following resection in Stage IB non-small cell lung cancer (NSCLC): Report of Cancer and Leukemia Group B (CALGB) Protocol 9633. Proc Am Soc Clin Oncol 2004, 23:619, abstract no. 7019.

29. Pradier O, Rave-Frank M, Schmidberger $\mathrm{H}$, et al: Effects of paclitaxel in combination with radiation on human head and neck cancer cells (ZMK1), cervical squamous cell carcinoma (CaSki), and breast adenocarcinoma cells (MCF-7). J Cancer Res Clin Oncol 1999, 125(1):20-7.

30. Britten RA, Perdue S, Opoku J, et al: Taxol is preferentially cytotoxic to human cervical tumor cells with low Raf-1 kinase activity: implications for Taxol-based chemoradiation regimens. Radiother Oncol 1998, 48:329-34.

31. Chen MD, Paley PJ, Potish RA, et al: Phase I trial of Taxol as radiation sensitizer with Cisplatin in advanced cervical cancer. Gynecol Oncol 1997, 67:131-6.

32. Vogt HG, Martin T, Kolotas C, et al: Simultaneous Taxol and radiotherapy: initial clinical experience in lung cancer and other malignancies. Semin Oncol 1997, 24:S12-101-S12-105.

33. Papadimitriou CA, Sarris K, Moulopoulos LA, et al: Phase II trial of Taxol and Cisplatin in metastatic and recurrent carcinoma of the cervix. J Clin Oncol 1999, 17:761-6.

34. Kudelka AP, Winn R, Edwards CL, Downey G, et al: Activity of Taxol in advanced or recurrent squamous cell cancer of the cervix. Clin Cancer Res 1996, 2:1285-8.

35. Moore DH, Blessing JA, McQuellon RP, et al: Phase III study of cisplatin with or without paclitaxel in stage IVB, recurrent, or persistent squamous cell carcinoma of the cervix: a gynecologic oncology group study. J Clin Oncol 2004, 22(15):3113-9.

36. Cancer Therapy Evaluation Program: Common toxicity criteria, version 2.0. Bethesda, Md.: National Cancer Institute 1998.
37. Rose PG, Bundy BN: Chemoradiation for Locally Advanced Cervical Cancer: Does It Help? J Clin Oncol 2002, 20(4):891-893.

38. Lee MY, Wu HG, Kim K, et al: Concurrent radiotherapy with paclitaxel/ carboplatin chemotherapy as a definitive treatment for squamous cell carcinoma of the uterine cervix. Gynecol Oncol 2007, 104(1):95-9.

39. Pignata S, Frezza P, Tramontana S, et al: Phase I study with weekly cisplatin-paclitaxel and concurrent radiotherapy in patients with carcinoma of the cervix uteri. Ann Oncol 2000, 11(4):455-9.

40. De Vos FY, Bos AM, Gietema JA, et al: Paclitaxel and carboplatin concurrent with radiotherapy for primary cervical cancer. Anticancer Res 2004, 24(1):345-8.

41. Rao GG, Rogers P, Drake RD, et al: Phase I clinical trial of weekly paclitaxel, weekly carboplatin, and concurrent radiotherapy for primary cervical cancer. Gynecol Oncol 2005, 96(1):168-72.

42. DiSilvestro PA, Walker JL, Morrison A, et al: Gynecologic Oncology Group. Radiation therapy with concomitant paclitaxel and cisplatin chemotherapy in cervical carcinoma limited to the pelvis: a phase 1/II study of the Gynecologic Oncology Group. Gynecol Oncol 2006, 103(3):1038-42.

43. Argenta PA, Ghebre R, Dusenbery KE, et al: Radiation therapy with concomitant and adjuvant cisplatin and paclitaxel in high-risk cervical cancer: long-term follow-up. Eur J Gynaecol Oncol 2006, 27(3):231-5.

44. Miglietta L, Franzone P, Centurioni MG, et al: A phase II trial with cisplatinpaclitaxel cytotoxic treatment and concurrent external and endocavitary radiation therapy in locally advanced or recurrent cervical cancer. Oncology 2006, 70(1):19-24.

45. Lanciano R, Calkins A, Bundy BN, et al: Randomized comparison of weekly cisplatin or protracted venous infusion of fluorouracil in combination with pelvic radiation in advanced cervix cancer: a gynecologic oncology group study. J Clin Oncol 2005, 23(33):8289-95.

46. Thomas G, Dembo A, Ackerman I, et al: A randomized trial of standard versus partially hyperfractionated radiation with or without concurrent 5-fluorouracil in locally advanced cervical cancer. Gynecol Oncol 1988, 69:137-145.

47. Wong LC, Ngan HYS, Cheung ANY, et al: Chemoradiation and adjuvant chemotherapy in cervical cancer. J Clin Oncol 1999, 17:2055-2060.

48. Lorvidhaya V, Chitapanarux I, Sangruchi S, et al: Concurrent mitomycin C, 5-fluorouracil, and radiotherapy in the treatment of locally advanced carcinoma of the cervix: a randomized trial. Int J Radiation Oncology Biol. Phys 2003, 55:1226-1232.

49. Rakovitch E, Flyes AW, Pintilie M, Leung PM: Role of mitomycin C in the development of late bowel toxicity following chemoradiation for locally advanced carcinoma of the cervix. Int J Radiat Oncol Biol Phys 1997, 38:979-987.

50. Pattaranutaporn P, Thirapakawong C, Chansilpa Y, et al: Phase II study of concurrent gemcitabine and radiotherapy in locally advanced stage IIIIB cervical carcinoma. Gynecol Oncol 2001, 81:404-407.

51. Umanzor J, Aguiluz M, Pineda C, et al: Concurrent cisplatin/gemcitabine chemotherapy along with radiotherapy in locally advanced cervical carcinoma: A phase II trial. Gynecol Oncol 2006, 100:70-75.

52. Swisher EM, Swensen RE, Greer B, et al: Weekly gemcitabine and cisplatin in combination with pelvic radiation in the primary therapy of cervical cancer: A phase I trial of the puget sound oncology consortium. Gynecol Oncol 2006, 101:429-435.

53. Duenas-Gonzalez A, Cetina-Perez L, Lopez-Graniel C, et al: Pathologic response and toxicity assessment of chemoradiotherapy with cisplatin versus cisplatin plus gemcitabine in cervical cancer: a randomized phase II study. Int J Radiation Oncology Biol Phys 2005, 61:817-823.

54. Micheletti E, LaFace B, Bianchi E, et al: Continuous infusion of carboplatin during conventional radiotherapy treatment in advanced squamous carcinoma of the cervix uteri IIB-IIB (UICC). A phase I/II and pharmacokinetic study. Am J Clin Oncol 1997, 20:613-620.

55. Muderspach LI, Curtin JP, Roman LD, et al: Carboplatin as a radiation sensitizer in locally advanced cervical cancer: a pilot study. Gynecol Oncol 1997, 65:336-342.

56. Corn BW, Hernandez E, Anderson L, et al: Phase $1 / / l$ study of concomitant irradiation and carboplatin for locally advanced carcinoma of the uterine cervix: An interim report. Am J Clin Oncol 1996, 19:317-321.

57. Duenas-Gonzalez A, Cetina L, Sanchez B, et al: A phase I study of carboplatin concurrent with radiation in FIGO stage IIIB cervix uteri carcinoma. Int J Radiat Oncol Biol Phys 2003, 56:1361-1365. 
58. Higgins RV, Naumann WR, Hall JB, Haake M: Concurrent carboplatin with pelvic radiation therapy in the primary treatment of cervix cancer. Gynecol Oncol 2003, 89:499-503.

59. Dubay RA, Rose PG, O'Malley DM, et al: Evaluation of concurrent and adjuvant carboplatin with radiation therapy for locally advanced cervical cancer. Gynecol Oncol 2004, 94:121-124.

60. Craighead PS, Pearcey R, Stuart G: A phase I/II evaluation of tirapazamine administered intravenously concurrent with cisplatin and radiotherapy in women with locally advanced cervical cancer. Int I Radiat Oncol Biol Phys 2000, 48:791-795.

61. Dunton CJ, King SA, Neufeld J, et al: Phase I study of topotecan and radiation therapy in advanced cervical cancer. Gynecol Oncol 2002, 85:185-188.

62. Padilla LA, Mitchell SK, Carson LF, et al: Phase I study of topotecan continuous infusion and weekly cisplatin with radiation therapy for locally advanced/recurrent cervical cancer. Proc Am Soc Clin Oncol 2005, 23:486s.

63. Morris $\mathrm{M}$, Blessing JA, Monk BJ, et al: Phase II study of cisplatin and vinorelbine in squamous cell carcinoma of the cervix: a gynecologic oncology group study. J Clin Oncol 2004, 22:3340-3344.

64. Mundt AJ, Rotmensch J, Waggoner SE, et al: Phase I trial of concomitant vinorelbine, cisplatin, and pelvic irradiation in cervical carcinoma and other advanced pelvic malignancies. Gynecol Oncol 2004, 92:801-805.

65. Green JA, Kirwan JM, Tierney JF, et al: Survival and recurrence after concomitant chemotherapy and radiotherapy for cancer of the uterine cervix: a systematic review and meta-analysis. Lancet 2001, 358(9284):781-6.

66. Green J, Kirwan J, Tierney J, et al: Concomitant chemotherapy and radiation therapy for cancer of the uterine cervix. Cochrane Database Syst Rev 2005, 20(3).

67. Kim YB, Cho JH, Keum KC, et al: Concurrent chemoradiotherapy followed by adjuvant chemotherapy in uterine cervical cancer patients with highrisk factors. Gynecol Oncol 2007, 104(1):58-63.

68. Lee JW, Kim BG, Lee SJ, et al: Preliminary results of consolidation chemotherapy following concurrent chemoradiation after radical surgery in high-risk early-stage carcinoma of the uterine cervix. Clin Oncol ( $R$ Coll Radiol) 2005, 17(6):412-7.

doi:10.1186/1748-717X-5-84

Cite this article as: Geara et al:: A phase II randomized trial comparing radiotherapy with concurrent weekly cisplatin or weekly paclitaxel in patients with advanced cervical cancer. Radiation Oncology 2010 5:84.

\section{Submit your next manuscript to BioMed Central and take full advantage of:}

- Convenient online submission

- Thorough peer review

- No space constraints or color figure charges

- Immediate publication on acceptance

- Inclusion in PubMed, CAS, Scopus and Google Scholar

- Research which is freely available for redistribution

Submit your manuscript at www.biomedcentral.com/submit
Biomed Central 